\title{
Anomalous connection of the left posterior renal vein with the left ascending lumbar vein in a Japanese cadaver
}

\author{
H. Terayama1 ${ }^{1}$ S.-Q. Yi², S. Shoji ${ }^{3}$, O. Tanaka ${ }^{1}$, T. Kanazawa ${ }^{1}$, N. Kosemura ${ }^{1}$, M. Tamura ${ }^{1}$, \\ M. Sekiguchi ${ }^{1}$, M. Naito ${ }^{4}$, T. Akamatsu ${ }^{5}$, K. Sakabe ${ }^{1}$ \\ ${ }^{1}$ Department of Anatomy, Division of Basic Medicine, Tokai University School of Medicine, Tokai, Japan \\ ${ }^{2}$ Laboratory of Functional Morphology, Department of Frontier Health Science, Tokyo Metropolitan University, Tokyo, Japan \\ ${ }^{3}$ Department of Urology, Tokai University Hachioji Hospital, Tokai, Japan \\ ${ }^{4}$ Department of Anatomy, Aichi Medical University, Aichi, Japan \\ ${ }^{5}$ Department of Plastic and Cosmetic Surgery, Tokai University School of Medicine, Tokai, Japan
}

[Received 17 December 2014; Accepted 22 January 2015]

\begin{abstract}
A rare variation was found in one of the two left renal veins in a 94-year-old male cadaver undergoing routine dissection. The characteristic findings in the cadaver included, in addition to the primary left renal vein, the presence of a posterior left renal vein draining to the left ascending lumbar vein without communicating with the inferior vena cava and other renal veins. Variations in the number and arrangement of the vessels terminating in the renal veins are common, but to our knowledge, variation similar to our findings has not been previously reported. This variation may represent an immature form of the complicated development of the renal vessels. (Folia Morphol 2015; 74, 4: 544-547)
\end{abstract}

Key words: left ascending lumbar vein, left renal vein, posterior tributary, vascular development

\section{INTRODUCTION}

Knowledge of vascular variation of the kidneys is clinically important, particularly concerning surgical resection for patients with renal cell carcinoma, renal transplantation, vascular surgery, renal trauma, and radiological interventions. The anatomy and embryological development of the left renal vein (LRV) is more complicated than that of the right renal vein because of its complex embryogenesis and its collocated relationships with the abdominal aorta and superior mesenteric artery. Normally, the LRV arises from the kidney, transverses to the right side in front of the aorta, and drains into the inferior vena cava (IVC). In addition, various veins (e.g., lumber, testicular, ovarian, and adrenal) also terminate at the LRV.
Developmental venous anomalies of the LRV arise as circumaortic LRV (CLRV) or retroaortic LRV (RLRV) [2]. Several reports described the clinical significance of RLRVs or CLRVs (also called 'circumaortic venous rings' or 'renal collars') [10]. Connections to the IVC exist in both normal and abnormal renal veins. Moreover, the LRV is normally located in the retroperitoneal space in front of the renal artery. A retropelvic tributary (RPT) of the renal vein, also called the posterior primary tributary $[4,7,8]$, posterior renal vein [5], or supernumerary renal vein [5], is a common variation of the LRV. Prior reports described tributaries that communicated with the LRV, although the RPT does not drain into the IVC. Moreover, several normal and abnormal LRVs are connected to the lumber vein $[9,10]$.

Address for correspondence: Dr H. Terayama, Department of Anatomy, Division of Basic Medicine, Tokai University School of Medicine, 143 Shimokasuya, Isehara-si, Kanagawa 259-1193, Japan, tel: +81-463-931121 (ext: 2513), e-mail: terahaya@tokai-u.jp 
However, to our knowledge, neither clinical nor gross anatomical reports of connections between the LRV and left ascending lumbar vein (LALV) without communication with other LRVs are present in the literature. This paper reports a connection between the LRV and LALV without communication with other LRVs in an adult cadaver. In addition to describing and illustrating this case, the clinical medicine and embryological development of the LRV and IVC are discussed.

\section{CASE REPORT}

This case was conducted on a 94-year-old Japanese adult male cadaver, which was selected from the bodies used for research and gross anatomy practice at Tokai University School of Medicine in the year 2013. The cadaver was fixated using $10 \%$ formaldehyde, and it was free of obvious diseases of the kidneys and related vessels. Gross dissection was performed using the customary procedure. The anatomical relationship between the LRV and IVC was specifically observed. For this purpose, we removed the remaining branches of the coeliac trunk and the branches of the upper and lower mesenteric arteries and carefully examined the structures.

The left renal vessels consisted of 2 arteries and 2 veins. The anterior LRV (aLRV) (approximately $17 \mathrm{~mm}$ wide), coursing anterior to the aorta and drained into the IVC at the level between the second and third lumbar vertebrae (i.e., normal position). Meanwhile, the posterior LRV (pLRV) (the diameter of the pLRV near the LALV was approximately $8 \mathrm{~mm}$ ) passed behind the aLRV and drained into the LALV without communicating grossly with other veins (particularly the aLRV) at the level of the third lumbar vertebrae, also called the RPT (Fig. 1A-C). The torn portion of pLRV is the artifactual error (Fig. 1B). The LALV ran upward into the chest region and drained into the azygos vein (Fig. 1B, C). The hemiazygos vein was absent (Fig. 1C). The left testicular vein was accompanied by the left testicular artery and drained into the aLRV (Fig. 1A). The left suprarenal vein passed obliquely downward and drained into the aLRV (Fig. 1A). Other arteries and veins displayed normal courses.

\section{DISCUSSION}

In this paper, a connection between the LRV and LALV without communication with the aLRV in a cadaver was described. The ascending lumbar veins are paired and they connect the common iliac vein, approximately 4 pairs of lumbar veins, and the iliolumbar veins. They ascend deeply into the psoas major muscle on to the medial portions of the lumber transverse processes to join with the subcostal veins, which drain into the azygos vein on the right side and hemiazygos vein on the left. Concerning the relationship between the LRV and lumber veins in normal anatomic studies, Yao et al. [9] and Raheem et al. [6] reported that the incidence of a communicating vein between the LRV and the LALV/hemiazygos venous system is approximately $90 \%$ based on prior anatomic reports. Concerning common variations of LRVs, Yi et al. [10] reported anastomoses between the CLRV/RLRV/RPT and the ascending lumbar veins and/or second and third lumbar veins. In this present study, the pLRV was connected to the LALV. An RPT is frequently observed, and its incidence in various cadaveric studies has been reported as $30.0 \%$ [3], $30.4 \%$ [5], and $46.4 \%$ [7]. Regarding the RPT, the left side differs from the right side in that the IVC is usually absent. In addition, the existence of communicating veins between the LRV and retroperitoneal veins, such as the lumbar, ascending lumbar, capsular, and hemiazygos, has been reported. However, in all cases of RPTs, irrespective of whether a connection exists between the LRV and retroperitoneal veins, the pLRV drains into the aLRV $[5,6]$. Thus, the lack of a connection between the pLRV and aLRV in the present case represents a rare finding.

During embryological development, the subcardinal and supracardinal veins form a vascular ring around the aorta (circumaortic ring) with several transverse anastomotic channels. The ventral part of the circumaortic ring persists as the usual LRV [9]. Variations in this developmental process explain the formation of the CLRV/RLRV $[9,10]$. In RPTs, the ventral part of the circumaortic ring persists, and the dorsal part of the circumaortic ring is connected to the aLRV. It is likely that the communicating vein between LRV and LALV is a remnant of the anastomotic channels between the subcardinal and supracardinal veins. Thus, the lack of a communicating vein between the pLRV and aLRV can be explained by this developmental process.

Clinically, an understanding of renal vein variation is important in cases of surgical resection of the kidneys for patients with renal cell carcinoma, renal transplantation, vascular surgery, the treatment of renal trauma, and radiological interventions. In patients with progressive renal cell carcinoma, tumour 


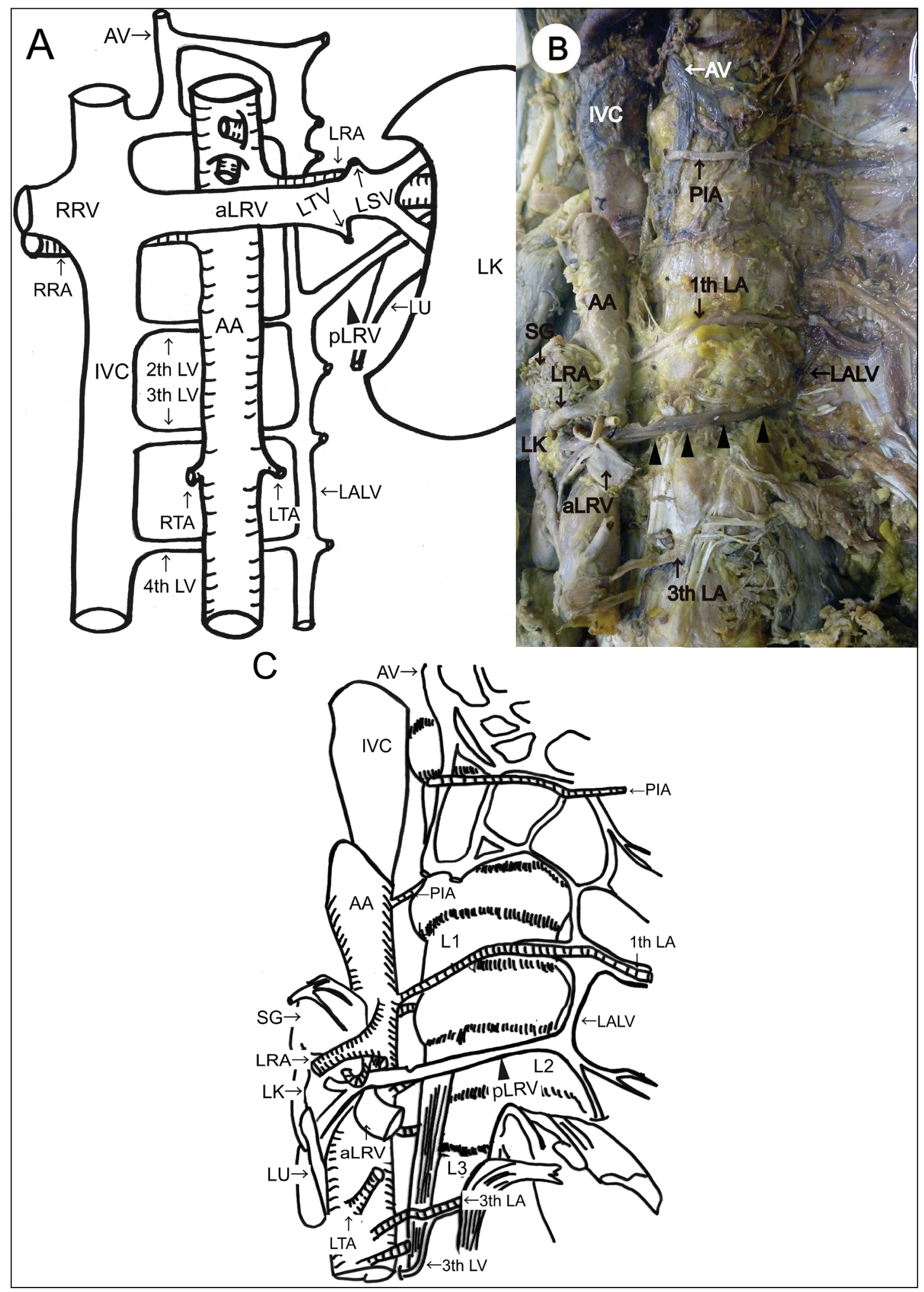

Figure 1. Schematic illustration (A) of the vasculature prior to reversed of the left kidney, a photograph (B) and a schematic illustration (C) of the posterior left renal vein. Schematic illustration (A) shows the organ in the original position in the body. The photograph (B) and schematic illustration (C) were taken from the left side of the body, and the kidney was reversed from the right to the left. The black arrowheads indicate the posterior left renal vein (pLRV). The pLRV passed behind the kidney and then drained into the left ascending lumbar vein without connecting to the inferior vena cava and anterior left renal vein (aLRV); AA — abdominal aorta; AV — azygos vein; IVC — inferior vena cava; PIA — posterior intercostal artery; LA — lumber artery; LALV — left ascending lumbar vein; LK — left kidney; LRA — left renal artery; LTA — left testicular artery; LTV — left testicular vein; LSV — left suprarenal vein; LV — lumber vein; LU — left ureter; RRA — right renal artery; RRV — right renal vein; RTA — right testicular artery; SG — suprarenal gland. 
thrombosis in the LRV should be targeted for complete resection [1]. Therefore, a pre- and intra-operative understanding of renal vein variation would help to ensure the complete resection of tumour thrombosis in the LRV [1]. Thus, an understanding of renal vein variation is clinically essential.

\section{ACKNOWLEDGEMENTS}

The authors thank Ms. Kyoko Endo and Ms. Yuko Furuya (All Tokai University School of Medicine, Kanagawa, Japan) for excellent secretarial support, and Ms. Chinami Tarumi, Ms. Junko Higashigawa, Mr. Gaku Yamazaki and Mr. Yudai Suzuki (All Tokai University School of Medicine, Kanagawa, Japan) for excellent technical support. This work was supported by Grant-in-Aid for Scientific Research (JSPS KAKENHI Grant Numbers C26460809, C25340050) from the Japan Ministry of Education, Culture, Sports, Science and Technology. The funders had no role in study design, data collection and analysis, decision to publish, or preparation of the manuscript.

\section{REFERENCES}

1. Lawindy SM, Kurian $T$, Kim T, Mangar D, Armstrong PA, Alsina AE, Sheffield C, Sexton WJ, Spiess PE (2012) Impor- tant surgical considerations in the management of renal cell carcinoma (RCC) with inferior vena cava (IVC) tumor thrombus. BJU Int, 110: 926-939.

2. Martinez-Almagro A, Almenar Garcia V, Martinez Sanjuan V, Hernandez Gil de Tejada T, Lorente Montalvo P (1992) Retroaortic left renal vein: a report of six cases. Surg Radiol Anat, 14: 361-366.

3. Merklin RJ, Mitchels NA (1958) The variant renal and suprarenal blood supply with data on the inferior phrenic, ureteral and gonadal arteries: a statistical analysis based on 185 dissections and review of the literature. J Int Coll Surg, 29: 41-76.

4. Nayak SB, Sirasanagandla SR, Shetty SD, Kumar N (2013) Multiple vascular variations at the vicinity of the left kidney. Anat Sci Int, 88: 230-233.

5. Okamoto K (1990) The posterior renal vein (new definition), together with its morphological significance. Okajimas Folia Anat Jpn, 67: 203-217.

6. Raheem OA, O'Brien M, Glacken P, Mohan P, Hickey DP (2008) A review of the anatomical variations of the posterior tributaries of the left renal vein. Ir J Med Sci, 177: 59-62.

7. Satyapal KS (1995) Classification of the drainage patterns of the renal veins. J Anat, 186: 329-333.

8. Satyapal KS, Kalideen JM, Haffejee AA, Singh B, Robbs JV (1999) Left renal vein variations. Surg Radiol Anat, 21:77-81.

9. Yao Y, Okada Y, Yamato M, Ohtomo K (2003) Communicating vein between the left renal vein and left ascending lumber vein: incidence and significance on abdominal CT. Radiat Med, 21: 252-257.

10. Yi SQ, Ueno Y, Naito M, Ozaki N, Itoh M (2012) The three most common variations of the left renal vein: a review and meta-analysis. Surg Radiol Anat, 34: 799-804. 